\title{
TAUBERIAN THEOREMS FOR THE LAPLACE-STIELTJES TRANSFORM
}

\author{
C. J. K. BATTY
}

\begin{abstract}
Let $\alpha:[0, \infty) \rightarrow \mathbf{C}$ be a function of locally bounded variation, with $\alpha(0)=0$, whose Laplace-Stieltjes transform $g(z)=\int_{0}^{\infty} e^{-z t} d \alpha(t)$ is absolutely convergent for $\operatorname{Re} z>0$. Let $E$ be the singular set of $g$ in $i \mathbf{R}$, and suppose that $0 \notin E$. Various estimates for lim sup $\sin _{t \rightarrow \infty}|\alpha(t)-g(0)|$ are obtained. In particular, $\alpha(t) \rightarrow g(0)$ as $t \rightarrow \infty$ if
\end{abstract}

$$
\begin{aligned}
& \text { (i) } E \text { is null, } \\
& \text { (ii) } \sup _{y \in E} \sup _{t>0}\left|\int_{0}^{t} e^{-i y s} d \alpha(s)\right|<\infty, \\
& \text { (iii) } \lim _{\delta \downarrow 0} \limsup _{t \rightarrow \infty} \sup _{t-\delta \leq s \leq t}|\alpha(s)-\alpha(t)|=0 .
\end{aligned}
$$

This result contains Tauberian theorems for Laplace transforms, power series, and Dirichlet series.

\section{INTRODUCTION}

There have recently appeared several theorems of Tauberian type concerning the convergence of power series, Dirichlet series, and Laplace transforms on the boundaries of their natural domains of convergence $[1,2,5,7,8,11]$. Up to a point, they can be formulated in the common language of Laplace-Stieltjes transforms.

If $\alpha:[0, \infty) \rightarrow \mathbf{C}$ is a function which is of locally bounded variation, and if

$$
g(z)=\int_{0}^{\infty} e^{-z t} d \alpha(t)
$$

is absolutely convergent whenever $\operatorname{Re} z>0$, then $g$ defines an analytic function in this region. Choosing $\alpha$ appropriately, one can arrange that $g$ is either a power series in $e^{-z}, g(z)=\sum_{n=0}^{\infty} a_{n} e^{-n z}$, a Dirichlet series $g(z)$ $=\sum_{n=1}^{\infty} a_{n} n^{-(1+z)}$, or a Laplace transform $g(z)=\int_{0}^{\infty} e^{-z t} f(t) d t$. If 0 is a regular point of $g$, a theorem of A. E. Ingham [3] gives an estimate for lim sup $\sin _{t \rightarrow \infty}|\alpha(t)-g(0)|$ in terms of the distance from 0 to the singular set of

Received by the editors February 6, 1989 and, in revised form, September 14, 1989.

1980 Mathematics Subject Classification (1985 Revision). Primary 44A10; Secondary 40E05, 47D05.

Key words and phrases. Tauberian theorem, Laplace-Stieltjes transform, Laplace transform, power series, Dirichlet series, $C_{0}$-semigroup. 
$g$ and other estimates on $\alpha$. If the singular set is empty and the other estimates can be obtained in the right form, it is possible to deduce that $\alpha(t) \rightarrow g(0)$ and similarly $\int_{0}^{t} e^{-i \eta s} d \alpha(s) \rightarrow g(i \eta)$ for all regular points $i \eta$. Thus if $g$ is either a Dirichlet series with bounded coefficients $\left(a_{n}\right)$ or the Laplace transform of a bounded function $f$, and $g$ has no singularities on the imaginary axis, then $\alpha(t) \rightarrow g(0)$, so that $\sum a_{n}$ converges or $\int_{0}^{\infty} f(s) d s$ converges conditionally.

These special cases of Ingham's theorem have recently been given elegant proofs, by means of contour integration, by Newman [7] for Dirichlet series, and by Korevaar [5] and Zagier [11] for Laplace transforms, in each case as part of an elementary proof of the Prime Number Theorem. The case of power series is more intricate, partly because there are always singularities on the circle of convergence, and partly because it is not possible to obtain exactly the conditions needed for Ingham's theorem. However, Katznelson and Tzafriri [4] obtained one result for power series with a single singularity, and this was extended to other cases in [1] by means of an adaptation of Newman's method. In [2] these ideas were used again for Laplace transforms, giving new Tauberian theorems. The papers $[1,2,4]$ all had specific applications to operator theory in mind.

The Tauberian theorems in $[1,2,5,7,11]$ have the following form. Let $\alpha$ be of a specified type, and suppose that the singular set $i E$ of $g$ on the imaginary axis is (Lebesgue) null, and that

$$
\sup _{y \in E} \sup _{t>0}\left|\int_{0}^{t} e^{-i y s} d \alpha(s)\right|<\infty
$$

Then

$$
\int_{0}^{t} e^{-i \eta s} d \alpha(s) \rightarrow g(i \eta) \quad \text { as } t \rightarrow \infty \text { for all } \eta \text { in } \mathbf{R} \backslash E .
$$

This result is not valid without some constraint on $\alpha$ apart from those mentioned in the second paragraph-there is an example in [2] where $\alpha$ is (vectorvalued) continuously differentiable and bounded, $\left\|\alpha^{\prime}(t)\right\| \leq c(1+t)$, and $E$ is empty, but (1.2) fails for all $\eta$.

This raises the question as to what conditions, in addition to nullity of $E$ and (1.1), ensure (1.2). It will be shown in Corollary 2.6 that it is sufficient that

$$
\lim _{\delta \downarrow 0} \limsup _{t \rightarrow \infty} \sup _{t-\delta \leq s \leq t}|\alpha(s)-\alpha(t)|=0 .
$$

This result can be deduced from the corresponding result for Laplace transforms of bounded functions [2]. Now (1.3) is satisfied for Dirichlet series with bounded coefficients as well as for Laplace transforms of bounded functions. However, for power series, the position is slightly different. Then (1.3) becomes

$$
\lim _{n \rightarrow \infty} a_{n}=0
$$

and it may not be clear, a priori, that (1.4) holds. Indeed, if (1.4) is known, then a classical theorem of M. Riesz (see [9]) shows that $\sum a_{n} z^{n}$ converges at all regular points. In the applications to operator theory in $[1,4]$, the important 
conclusion was that (1.4) follows from the assumptions that $\left(a_{n}\right)$ is bounded, $E$ is null, and (1.1) holds. The Tauberian theorem proved in [1], and hence the validity of (1.4), can be deduced from the corresponding result for Laplace transforms of bounded functions [2] (see $\$ 5$ ).

Apart from showing that (1.3) is a suitable condition for a Tauberian theorem for Laplace-Stieltjes transforms, we also give several other estimates on

$$
\limsup _{t \rightarrow \infty}\left|\int_{0}^{t} e^{-i \eta s} d \alpha(s)-g(i \eta)\right| .
$$

They take a different form to Ingham's estimate, and some of them are analogous to results of Ransford [8] for power series. An interesting consequence is an analogue of the theorem of Riesz, showing that conditional convergence of $\int_{0}^{\infty} e^{-i \eta t} d \alpha(t)$ for any value of $\eta$ implies conditional convergence for all regular points in (Corollary 2.5).

The main results are stated in $\S 2$ and proved in $\S 3$, and there follow interpretations for Laplace transforms, power series, and Dirichlet series. The last two sections contain some refinements of the stability theorems for operators obtained in [2,6]. Further developments of these may be found in [12].

I am very grateful to W. Arendt and T. J. Ransford for several valuable discussions on this subject and for their perceptive remarks, some of which have been included in the paper. I am also grateful to the referee for having pointed out a number of inaccuracies in the original version.

\section{THE MAIN THEOREMS}

Throughout, $\alpha$ will be a (strongly) measurable function on $[0, \infty)$, taking values in $\mathbf{C}$ (or, more generally, in any Banach space), and satisfying

$$
\int_{0}^{\infty} e^{-x t}|\alpha(t)| d t<\infty
$$

for each $x>0$. We shall consider the analytic functions

$$
\begin{gathered}
g(z)=z \int_{0}^{\infty} e^{-z s} \alpha(s) d s-\alpha(0) \quad(\operatorname{Re} z>0), \\
g_{t}(z)=e^{-z t} \alpha(t)+z \int_{0}^{t} e^{-z s} \alpha(s) d s-\alpha(0) \quad(z \in \mathbf{C}, t \geq 0) .
\end{gathered}
$$

Let

$$
E=\{y \in \mathbf{R}: i y \text { is a singular point of } g\} .
$$

Let $\eta$ be a fixed point in $\mathbf{R} \backslash E$. Our aim is to estimate

$$
G:=\limsup _{t \rightarrow \infty}\left|g_{t}(i \eta)-g(i \eta)\right|
$$


in terms of quantities depending on $\alpha, E$, and $\eta$, such as

$$
\begin{aligned}
M & :=\inf _{a \in \mathrm{C}} \limsup _{t \rightarrow \infty}|\alpha(t)-a|, \\
L & :=\lim _{\delta \downarrow 0} \limsup _{t \rightarrow \infty} \sup _{t-\delta \leq s \leq t}|\alpha(s)-\alpha(t)|, \\
N & :=\limsup _{s, t \rightarrow \infty}\left|\frac{\alpha(t)-\alpha(s)}{t-s}\right|, \\
\mu & :=\sup _{y \in E}\left|\frac{y}{y-\eta}\right| \\
d & :=\inf _{y \in E}|y-\eta| .
\end{aligned}
$$

Remarks. 1. If $E$ is empty, our results are still applicable-one should conventionally regard $\mu=0$ and $d=\infty$-and some of them are already known $[3,5,7,9]$. Otherwise the results are applicable only under assumptions on $\alpha$ which ensure that $L, M$, or $N$ is finite.

2. If $\alpha$ is of locally bounded variation, then partial integration gives

$$
g(z)=\int_{0}^{\infty} e^{-z s} d \alpha(s), \quad g_{t}(z)=\int_{0}^{t} e^{-z s} d \alpha(s),
$$

provided that the former integral is absolutely convergent whenever $\operatorname{Re} z>$ 0 . If, in addition, $\alpha$ is locally absolutely continuous, then there is a locally integrable function $f$ such that

$$
\begin{aligned}
\alpha(t) & =\alpha(0)+\int_{0}^{t} f(s) d s, \\
g(z) & =\int_{0}^{\infty} e^{-z s} f(s) d s, \\
g_{t}(z) & =\int_{0}^{t} e^{-z s} f(s) d s .
\end{aligned}
$$

3. Traditionally, a Tauberian theorem gives information about the limiting behaviour of $g_{t}(0)=\alpha(t)-\alpha(0)$. Our results give information about $g_{t}(i \eta)$ (where $\eta$ should be thought of as being nonzero-see Remark 5 at the end of this section) in terms of $\alpha$. However, if the results are applied with $\alpha(t)$ replaced by $\alpha_{\eta}(t):=g_{t}(i \eta)$ and $\eta$ replaced by $-\eta$, then they give information about the original $\alpha(t)$ in terms of $\alpha_{\eta}$. Note that when $\alpha$ is replaced by $\alpha_{\eta}$, then $g(z), g_{t}(z)$, and $E$ are replaced by $g(z+i \eta), g_{t}(z+i \eta)$, and $E-\eta$ respectively, while the value of $N$ is unchanged.

We now state the main results and make some further remarks about them. The proofs will be given in the next section, and the following three sections show how our theorems relate to known results.

Theorem 2.1. If $\alpha$ is bounded on $[\tau, \infty$ ) for some $\tau>0$ (so that $M<\infty$ ), then

$$
G \leq 2\left(\frac{2}{\pi}+\max (\mu, 1)\right) M .
$$


Theorem 2.2. Suppose that $\alpha$ is Lipschitz on $[\tau, \infty)$ for some $\tau>0$ (so that $N<\infty)$. Then

$$
G \leq \frac{2 N}{d}
$$

Hence, for any regular point i $\eta, \sup _{t>0}\left|g_{t}(i \eta)\right|<\infty$. If, in addition, $\alpha$ is bounded on $[\tau, \infty)$ (for example, if 0 is regular), then

$$
G \leq 2\left(\frac{2}{\pi} \min (\mu, 1)+\mu\right) M .
$$

Theorem 2.3. If $\alpha$ is bounded on $[\tau, \infty)$ for some $\tau>0$ (so that $M<\infty$ ), then

$$
G \leq 2\left(\frac{2}{\pi} \min (\mu, 1)+\mu\right) M+L .
$$

If $E$ is empty and $L$ is finite (but $M$ is possibly infinite), then $G \leq L$.

Theorem 2.4. Let $E^{\prime}$ be a closed subset of $E$ such that $E \backslash E^{\prime}$ is null and

$$
\sup _{y \in E \backslash E^{\prime}} \sup _{t>0}\left|g_{t}(i y)\right|<\infty .
$$

Let

$$
\mu^{\prime}=\sup _{y \in E^{\prime}}\left|\frac{y}{y-\eta}\right|
$$

(with $\mu^{\prime}=0$ if $E^{\prime}$ is empty). Then Theorems 2.1, 2.2, and 2.3 remain valid with $E$ and $\mu$ replaced by $E^{\prime}$ and $\mu^{\prime}$.

Corollary 2.5. Suppose that $\lim _{t \rightarrow \infty} g_{t}(i \zeta)$ exists for some $\zeta$ in $\mathbf{R}$. Then $\lim _{t \rightarrow \infty} g_{t}(i \eta)=g(i \eta)$ for all regular points $i \eta$.

Corollary 2.6. Suppose that $E$ is null, and

$$
\lim _{\delta \downarrow 0} \limsup _{t \rightarrow \infty} \sup _{t-\delta \leq s \leq t}|\alpha(s)-\alpha(t)|=0 .
$$

Then

$$
g_{t}(\text { in }) \rightarrow g(\text { in }) \text { as } t \rightarrow \infty
$$

for all regular points.

Remarks. 1. If $\mu \geq 1$ (that is, if $i \eta$ is close to a singular point of $g$ ), Theorem 2.1 is analogous to, but more general than, [8, Theorem 1.1], and it will be proved by refining the argument of [2, Lemma 3.1] along lines parallel to those of [8].

2. The last statement of Theorem 2.2 is clearly a special case of Theorem 2.3 , and we shall consider the case when $\alpha$ is Lipschitz in more detail in $\S 4$. However, we have included the statement here because Theorem 2.3 will be deduced from it. 
3. The estimates obtained in Theorems $2.1,2.2$, and 2.3 are not necessarily optimal. Let

$$
M^{*}=\limsup _{s, t \rightarrow \infty}|\alpha(s)-\alpha(t)| .
$$

A variation of the proofs in $\S 3$ (in particular, the estimates (3.1) and (3.2)) shows that $G \leq 2 M^{*} \max (\mu, 1)$ and $G \leq 2 M^{*} \mu+L$. If $\alpha$ is complex-valued, then $\sqrt{3} M \leq M^{*} \leq 2 M\left(M^{*}=2 M\right.$ if $\alpha$ is real-valued $)$, so these estimates are less sharp than Theorems 2.1 and 2.3. However, if $\alpha$ takes values in an arbitrary Banach space (so $M \leq M^{*} \leq 2 M$ ), the estimates in terms of $M^{*}$ may be sharper.

4. In the setting of Example 2.5b) of [2], let $\alpha$ be the bounded (vector-valued) function $\alpha(t)=T(t) y$. Then $E$ is empty, but $M^{*}=2 e^{-1}, M=e^{-1}=G$ for any value of $\eta$. Thus the estimate in Theorem 2.2 may fail if $\alpha$ is not Lipschitz. Similarly, Corollary 2.6 may fail if $(2.7)$ is not satisfied.

5. If 0 is a regular point, and we take $\eta=0$ (so that $\mu=1$ unless $E$ is empty), then it is easily seen that $G \leq M^{*} \leq 2 M$. For any $t>t_{0} \geq 0$,

$$
\begin{aligned}
\left|g_{t}(0)-g(0)\right| & =\lim _{x \downarrow 0}\left|g_{t}(0)-g(x)\right|=\left|\lim _{x \downarrow 0} \int_{0}^{\infty} x e^{-x s}(\alpha(t)-\alpha(s)) d s\right| \\
& \leq \limsup _{x \downarrow 0}\left\{x \int_{0}^{t_{0}}|\alpha(t)-\alpha(s)| d s+\int_{t_{0}}^{\infty} x e^{-x s}|\alpha(t)-\alpha(s)| d s\right\} \\
& \leq \sup _{s, t \geq t_{0}}|\alpha(t)-\alpha(s)| .
\end{aligned}
$$

6. If $\alpha$ is increasing and locally absolutely continuous, and $0 \notin E$, then $G=0$. For, $\alpha$ is given by (2.2) where $f \geq 0$ and the Pringsheim-Landau theorem [10, p. 117] shows that $f(t) e^{\lambda t}$ is integrable over $[0, \infty)$ for some $\lambda>0$. It is then immediate that $g_{t}(i \eta) \rightarrow g(i \eta)$.

7. The conclusion (2.8) of Corollary 2.6 "almost" implies condition (2.7) $(L=0)$. With $\alpha_{\zeta}(t)=g_{t}(i \zeta),(2.8)$ certainly implies that

$$
\lim _{\delta \downarrow 0} \limsup _{t \rightarrow \infty} \sup _{t-\delta \leq s \leq t}\left|\alpha_{\zeta}(s)-\alpha_{\zeta}(t)\right|=0
$$

for almost all $\zeta$. Under certain conditions, it follows from elementary estimates that (2.9) holds for all $\zeta$. Conversely, if (2.9) holds for some $\zeta$, and $E$ is null and (2.7) holds, then (2.8) holds (just apply Corollary 2.6 to $\alpha_{\zeta}$ ).

8. Ingham [3, Theorem I] showed that if $\alpha$ is of locally bounded variation then

$$
G \leq 2\left(1+\frac{3|z+i \eta|)}{d}\right) \Theta_{z}
$$

( $G \leq 2 \Theta_{z}$ if $E$ is empty) for any complex number $z$ such that

$$
\Theta_{z}:=\limsup _{t \rightarrow \infty}\left|e^{-t z} g_{t}(-z)\right|<\infty .
$$

When $\alpha$ is Lipschitz, (2.10) can be compared with (2.5), since $\Theta_{x-i \eta} \leq N / x$ $(x>0)$, so $(2.10)$ gives $G \leq 6 N / d$. 


\section{THE PROOFS}

The proofs of Theorems 2.1 and 2.2 both involve the ideas established in [2], but the estimates (3.1) and (3.2) below are more subtle, and the scaling function $h_{1}$ and the semicircle $\gamma_{1}$ of integration are more carefully chosen.

Proofs of Theorems 2.1 and 2.2. If $\alpha(t)=0$ whenever $t>\tau$, then $|\alpha|$ is integrable, $g$ is entire analytic, and it is trivial that $G=0$. Also, if $\alpha$ is constant, then $g=g_{t}=0$, so $G=0$. This permits us to make certain reductions. (Alternatively, we could perform the following estimates more carefully.)

Assume that $\alpha$ is bounded on $[\tau, \infty)$, and take $M^{\prime}>M$. We shall show that

$$
G \leq 2\left(\frac{2}{\pi}+\max (\mu, 1)\right) M^{\prime}
$$

Since $M^{\prime}$ is arbitrary, Theorem 2.1 will follow. By adding a constant to $\alpha$, we may assume that $\limsup _{t \rightarrow \infty}|\alpha(t)|<M^{\prime}$. Then, by increasing $\tau$, we may assume that $|\alpha(t)| \leq M^{\prime}$ whenever $t>\tau$. We may write $\alpha=\alpha_{1}+\alpha_{2}$, where $\alpha_{1}(t)=0$ whenever $t>\tau$, and $\left|\alpha_{2}(t)\right| \leq M^{\prime}$ whenever $t \leq \tau$. By our first observation, we may assume that $\alpha=\alpha_{2}$, so $|\alpha(t)| \leq M^{\prime}$ for all $t$, and we can arrange that $\alpha(0)=0$.

For $\operatorname{Re} z=x>0$,

$$
\begin{aligned}
\left|\left(g(z)-g_{t}(z)\right) e^{t z}\right| & =\left|-\alpha(t)+z \int_{t}^{\infty} e^{-(s-t) z} \alpha(s) d s\right| \\
& \leq M^{\prime}\left(1+\frac{|z|}{x}\right) .
\end{aligned}
$$

For $\operatorname{Re} z=x<0$,

$$
\begin{aligned}
\left|g_{t}(z) e^{t z}\right| & =\left|\alpha(t)+z \int_{0}^{t} e^{-(s-t) z} \alpha(s) d s\right| \\
& \leq M^{\prime}\left(1+\frac{|z|}{|x|}\right) \quad(x<0) .
\end{aligned}
$$

If $\eta=0$, then $G \leq 2 M$ (see Remark 5 at the end of $\S 2$ ), so we may assume that $\eta \neq 0$. Let $\lambda>0, \lambda \neq 1$, and let $\gamma_{1}$ be the circle $|z|=\lambda|z-i \eta|$. This is symmetrical about the $y$-axis, which it intersects at $i \xi_{+}$and $i \xi_{-}$, where $\xi_{ \pm}=\lambda \eta(\lambda \pm 1)^{-1}$. On $\gamma_{1}$,

$$
\begin{gathered}
\lambda(z-i \eta)=z e^{i \theta} \quad \text { for some } \theta, \\
z=\frac{i \lambda \eta}{\lambda-e^{i \theta}}=\frac{\lambda \eta(-\sin \theta+i(\lambda-\cos \theta))}{\left|\lambda-e^{i \theta}\right|^{2}}, \\
z-i \eta=\frac{i \eta e^{i \theta}}{\lambda-e^{i \theta}}, \quad \frac{d z}{d \theta}=\frac{-\lambda \eta e^{i \theta}}{\left(\lambda-e^{i \theta}\right)^{2}} .
\end{gathered}
$$

Let $\gamma_{1}^{+}$(resp. $\gamma_{1}^{-}$) be the part of $\gamma_{1}$ lying in the right (resp. left) half-plane. 
Thus

$$
\begin{aligned}
\left|\left(g(z)-g_{t}(z)\right) e^{t z}\right| \leq M^{\prime}\left(1+\frac{\left|\lambda-e^{i \theta}\right|}{|\sin \theta|}\right) & \left(z \in \gamma_{1}^{+}\right), \\
\left|g_{t}(z) e^{t z}\right| \leq M^{\prime}\left(1+\frac{\left|\lambda-e^{i \theta}\right|}{|\sin \theta|}\right) & \left(z \in \gamma_{1}^{-}\right) .
\end{aligned}
$$

Let

$$
h_{1}(z)=1-\frac{\lambda^{2}(z-i \eta)^{2}}{z^{2}} \text {. }
$$

For $z$ in $\gamma_{1}$,

$$
h_{1}(z)=1-e^{2 i \theta}, \quad\left|h_{1}(z)\right|=2|\sin \theta| .
$$

The semicircles $\gamma_{1}^{+}$and $\gamma_{1}^{-}$correspond to values of $\theta$ lying in intervals $I^{+}$ and $I^{-}$each of which is either $(0, \pi)$ or $(\pi, 2 \pi)$. Thus

$$
\begin{aligned}
& \left|\int_{\gamma_{1}^{+}} h_{1}(z)\left(g(z)-g_{t}(z)\right) \frac{e^{t(z-i \eta)}}{z-i \eta} d z\right| \\
& \quad \leq \int_{I^{+}} 2|\sin \theta| M^{\prime}\left(1+\frac{\left|\lambda-e^{i \theta}\right|}{|\sin \theta|}\right) \frac{\left|\lambda-e^{i \theta}\right|}{|\eta|} \frac{\lambda|\eta|}{\left|\lambda-e^{i \theta}\right|^{2}} d \theta \\
& \quad=2 M^{\prime} \lambda \int_{0}^{\pi}\left(\frac{\sin \theta}{\left(\lambda^{2}+1-2 \lambda \cos \theta\right)^{\frac{1}{2}}}+1\right) d \theta \\
& \quad=2 M^{\prime}(2 \min (\lambda, 1)+\lambda \pi) .
\end{aligned}
$$

Similarly,

$$
\left|\int_{y_{1}^{-}} h_{1}(z) g_{t}(z) \frac{e^{t(z-i \eta)}}{z-i \eta} d z\right| \leq 2 M^{\prime}(2 \min (\lambda, 1)+\lambda \pi) .
$$

Now, suppose that $\lambda>\max (\mu, 1)$. Then the interval between $\xi_{+}$and $\xi_{-}$ contains $\eta$ but does not contain 0 or any point of $E$. Let $\gamma_{2}$ be a fixed suitable path from $i \xi_{+}$to $i \xi_{-}$lying in the left half-plane and in a suitable domain of analyticity for $g$. (Note that $\gamma_{2}$ depends on $\lambda$, but not on $t$.) Then $\gamma_{1}^{+}$and $\gamma_{2}$ form a closed contour around $i \eta$ in a simply-connected domain not including 0 , and $\gamma_{1}^{-}$and $\gamma_{2}$ form a closed contour including neither 0 nor $i \eta$. By Cauchy's Theorem,

$$
\begin{aligned}
g(i \eta)-g_{t}(i \eta)= & \frac{1}{2 \pi i} \int_{\gamma_{1}^{+} \cup \gamma_{2}} h_{1}(z)\left(g(z)-g_{t}(z)\right) \frac{e^{t(z-i \eta)}}{z-i \eta} d z \\
= & \frac{1}{2 \pi i} \int_{\gamma_{1}^{+}} h_{1}(z)\left(g(z)-g_{t}(z)\right) \frac{e^{t(z-i \eta)}}{z-i \eta} d z \\
& +\frac{1}{2 \pi i} \int_{\gamma_{2}} h_{1}(z) g(z) \frac{e^{t(z-i \eta)}}{z-i \eta} d z \\
& -\frac{1}{2 \pi i} \int_{\gamma_{1}^{-}} h_{1}(z) g_{t}(z) \frac{e^{t(z-i \eta)}}{z-i \eta} d z .
\end{aligned}
$$


Since

$$
\int_{\gamma_{2}} h_{1}(z) g(z) \frac{e^{t(z-i \eta)}}{z-i \eta} d z \rightarrow 0 \quad \text { as } \quad t \rightarrow \infty
$$

by the Bounded Convergence Theorem, it follows that

$$
\limsup _{t \rightarrow \infty}\left|g(i \eta)-g_{t}(i \eta)\right| \leq 2\left(\frac{2}{\pi}+\lambda\right) M^{\prime} .
$$

Letting $M^{\prime} \downarrow M$ and $\lambda \downarrow \max (\mu, 1)$, Theorem 2.1 follows.

Now, we turn to the proof of Theorem 2.2. Let $N^{\prime}>N$. As before, we may assume that $|\alpha(s)-\alpha(t)| \leq N^{\prime}|s-t|$ for all $s, t$, so that (2.2), (2.3), and (2.4) hold for some $f$ with $|f(s)| \leq N^{\prime}$ for all $s$. Now the estimate $G \leq 2 N^{\prime} / d$ follows from the method of Korevaar [5] and Zagier [11] applied with $f(t)$ replaced by $e^{-i \eta t} f(t)$ (see also [2, Lemma 3.1]). It is immediate that $\sup _{t>0}\left|g_{t}(i \eta)\right|<\infty$ for any regular point $i \eta$, and hence that $\alpha$ is bounded if 0 is a regular point.

Now, assume in addition that $\alpha$ is bounded on $[\tau, \infty)$. Take $M^{\prime}>M$. As before, we may assume that $|\alpha(t)| \leq M^{\prime}$ for all $t$. In view of Theorem 2.1, we may suppose that $\mu<1$. Take $\lambda$ such that $\mu<\lambda<1$. The interval between $\xi_{+}$and $\xi_{-}$contains 0 and every point of $E$, but it does not contain $\eta$. Take $R>|\eta|(1-\lambda)^{-1}$, and let $\gamma_{0}$ be the circle $|z-i \eta|=R$ and $\gamma_{0}^{+}$and $\gamma_{0}^{-}$be the semicircles in the right and left half-planes, respectively. On $\gamma_{0}$,

$$
z=i \eta+R e^{i \theta},
$$

$$
\text { 3.3) } \begin{aligned}
\left|\left(g(z)-g_{t}(z)\right) e^{t z}\right| & =\left|\int_{t}^{\infty} e^{-(s-t) z} f(s) d s\right| \leq \frac{N^{\prime}}{R \cos \theta} \quad\left(z \in \gamma_{0}^{+}\right), \\
\left|g_{t}(z) e^{-t z}\right| & =\left|\int_{0}^{t} e^{-(s-t) z} f(s) d s\right| \leq \frac{N^{\prime}}{R|\cos \theta|} \quad\left(z \in \gamma_{0}^{-}\right) .
\end{aligned}
$$

Let

$$
h_{0}(z)=1+\frac{(z-i \eta)^{2}}{R^{2}}
$$

On $\gamma_{1}$,

$$
\left|h_{0}(z)\right| \leq 1+\frac{\eta^{2}}{R^{2}(1-\lambda)^{2}}
$$

so

$$
\begin{array}{r}
\left|\int_{\gamma_{1}^{+}} h_{0}(z) h_{1}(z)\left(g(z)-g_{t}(z)\right) \frac{e^{t(z-i \eta)}}{z-i \eta} d z\right| \leq 2 M^{\prime} \lambda(2+\pi)\left(1+\frac{\eta^{2}}{R^{2}(1-\lambda)^{2}}\right), \\
\left|\int_{\gamma_{1}^{-}} h_{0}(z) h_{1}(z) g_{t}(z) \frac{e^{t(z-i \eta)}}{z-i \eta} d z\right| \leq 2 M^{\prime} \lambda(2+\pi)\left(1+\frac{\eta^{2}}{R^{2}(1-\lambda)^{2}}\right) .
\end{array}
$$

On $\gamma_{0}$, where $z=i \eta+R e^{i \theta}$,

$$
\left|h_{0}(z)\right|=2|\cos \theta|, \quad\left|h_{1}(z)\right| \leq 1+\frac{\lambda^{2} R^{2}}{(R-|\eta|)^{2}} .
$$


Thus

$$
\begin{aligned}
& \left|\int_{\gamma_{0}^{+}} h_{0}(z) h_{1}(z)\left(g(z)-g_{t}(z)\right) \frac{e^{t(z-i \eta)}}{z-i \eta} d z\right| \\
& \quad \leq \int_{-\pi / 2}^{\pi / 2} 2 \cos \theta\left(1+\frac{\lambda^{2} R^{2}}{(R-|\eta|)^{2}}\right) \frac{N^{\prime}}{R \cos \theta} d \theta=\frac{2 \pi N^{\prime}}{R}\left(1+\frac{\lambda^{2} R^{2}}{(R-|\eta|)^{2}}\right), \\
& \left|\int_{\gamma_{0}^{-}} h_{0}(z) h_{1}(z) g_{t}(z) \frac{e^{t(z-i \eta)}}{z-i \eta} d z\right| \leq \frac{2 \pi N^{\prime}}{R}\left(1+\frac{\lambda^{2} R^{2}}{(R-|\eta|)^{2}}\right) .
\end{aligned}
$$

Let $\gamma_{2}$ be the union of suitable paths in the left half-plane, and in a suitable domain of analyticity for $g$, joining $i(\eta+R)$ to $i \xi_{+}$and $i \xi_{-}$to $i(\eta-R)$ (or vice versa if $\eta<0$ ). (Note that $\gamma_{2}$ depends on $\lambda$ and $R$, but not on $t$.) Then $\gamma_{0}^{+}, \gamma_{1}^{+}$, and $\gamma_{2}$ (with appropriate orientations) form a closed contour around $i \eta$ in a simply-connected domain not including 0 , and $\gamma_{0}^{-}, \gamma_{1}^{-}$, and $\gamma_{2}$ form a closed contour including neither 0 nor $i \eta$. By Cauchy's Theorem,

$$
\begin{aligned}
g(i \eta)-g_{t}(i \eta)= & \frac{1}{2 \pi i} \int_{\gamma_{0}^{+} \cup \gamma_{1}^{+} \cup \gamma_{2}} h_{0}(z) h_{1}(z)\left(g(z)-g_{t}(z)\right) \frac{e^{t(z-i \eta)}}{z-i \eta} d z \\
= & \frac{1}{2 \pi i} \int_{\gamma_{0}^{+} \cup \gamma_{1}^{+}} h_{0}(z) h_{1}(z)\left(g(z)-g_{t}(z)\right) \frac{e^{t(z-i \eta)}}{z-i \eta} d z \\
& +\frac{1}{2 \pi i} \int_{\gamma_{2}} h_{0}(z) h_{1}(z) g(z) \frac{e^{t(z-i \eta)}}{z-i \eta} d z \\
& -\frac{1}{2 \pi i} \int_{\gamma_{0}^{-} \cup \gamma_{1}^{-}} h_{0}(z) h_{1}(z) g_{t}(z) \frac{e^{t(z-i \eta)}}{z-i \eta} d z .
\end{aligned}
$$

Since

$$
\int_{\gamma_{2}} h_{0}(z) h_{1}(z) g(z) \frac{e^{t(z-i \eta)}}{z-i \eta} d z \rightarrow 0 \quad \text { as } t \rightarrow \infty
$$

by the Bounded Convergence Theorem,

$$
\begin{aligned}
\underset{t \rightarrow \infty}{\limsup }\left|g(i \eta)-g_{t}(i \eta)\right| \\
\quad \leq 2\left(\frac{2}{\pi}+1\right) M^{\prime} \lambda\left(1+\frac{\eta^{2}}{R^{2}(1-\lambda)^{2}}\right)+\frac{2 N^{\prime}}{R}\left(1+\frac{\lambda^{2} R^{2}}{(R-|\eta|)^{2}}\right) .
\end{aligned}
$$

Letting $R \rightarrow \infty, \lambda \downarrow \mu$, and $M^{\prime} \downarrow M$, Theorem 2.2 follows.

Proof of Theorem 2.3. We assume that $L$ is finite. Take $\delta>0$ such that $\delta|\eta|<2 \pi$, and put

$$
f_{\delta}(t)= \begin{cases}\alpha(t)-\alpha(0) & (0 \leq t \leq \delta) \\ \alpha(t)-\alpha(t-\delta) & (t \geq \delta)\end{cases}
$$


Then $f_{\delta}$ is bounded on $[\tau+\delta, \infty)$, and its Laplace transform is

$$
\begin{aligned}
\int_{0}^{\infty} e^{-z t} f_{\delta}(t) d t & =\int_{0}^{\infty} \alpha(t) e^{-z t} d t-\alpha(0) \int_{0}^{\delta} e^{-z t} d t-\int_{\delta}^{\infty} \alpha(t-\delta) e^{-z t} d t \\
& =\int_{0}^{\infty} \alpha(t) e^{-z t} d t-\frac{\alpha(0)}{z}\left(1-e^{-\delta z}\right)-e^{-\delta z} \int_{0}^{\infty} \alpha(t) e^{-z t} d t \\
& =\left(1-e^{-\delta z}\right)\left\{\int_{0}^{\infty} \alpha(t) e^{-z t} d t-\frac{\alpha(0)}{z}\right\} \\
& =\left(\frac{1-e^{-\delta z}}{z}\right) g(z) .
\end{aligned}
$$

Thus the singular set of this function is contained in $i E$. Moreover, if $\eta \neq 0$ and $t>\delta$, hen

$$
\begin{aligned}
\int_{0}^{t} e^{-i \eta s} f_{\delta}(s) d s= & \int_{0}^{t} e^{-i \eta s} \alpha(s) d s-\alpha(0) \int_{0}^{\delta} e^{-i \eta s} d s-\int_{\delta}^{t} e^{-i \eta s} \alpha(s-\delta) d s \\
= & \left(1-e^{-i \eta \delta}\right)\left\{\int_{0}^{t} e^{-i \eta s} \alpha(s) d s-\frac{\alpha(0)}{i \eta}\right\} \\
& +e^{-i \eta \delta} \int_{t-\delta}^{t} e^{-i \eta s} \alpha(s) d s \\
= & \left(\frac{1-e^{-i \eta \delta}}{i \eta}\right)\left(g_{t}(i \eta)-e^{-i \eta t} \alpha(t)\right) \\
& +e^{-i \eta \delta} \int_{t-\delta}^{t} e^{-i \eta s} \alpha(s) d s \\
= & \left(\frac{1-e^{-i \eta \delta}}{i \eta}\right) g_{t}(i \eta)+e^{-i \eta \delta} \int_{t-\delta}^{t} e^{-i \eta s}(\alpha(s)-\alpha(t)) d s .
\end{aligned}
$$

Let

$$
\alpha_{\delta}(t)=\int_{0}^{t} f_{\delta}(s) d s=\int_{t-\delta}^{t} \alpha(s) d s-\delta \alpha(0) .
$$

Then $\alpha_{\delta}$ is Lipschitz and bounded on $[\tau+\delta, \infty)$, so Theorem 2.2 gives:

$$
\begin{aligned}
\limsup _{t \rightarrow \infty}\left|\left(\frac{1-e^{-i \eta \delta}}{i \eta}\right)\left(g_{t}(i \eta)-g(i \eta)\right)+e^{-i \eta \delta} \int_{t-\delta}^{t} e^{-i \eta s}(\alpha(s)-\alpha(t)) d s\right| \\
\quad \leq 2\left(\frac{2}{\pi} \min (\mu, 1)+\mu\right) M_{\delta},
\end{aligned}
$$

where

$$
\begin{aligned}
M_{\delta} & =\inf _{a \in \mathbf{C}} \limsup _{t \rightarrow \infty}\left|\alpha_{\delta}(s)-a\right| \\
& =\inf _{a \in \mathbf{C}} \limsup _{t \rightarrow \infty}\left|\int_{t-\delta}^{t} \alpha(s) d s-\delta \alpha(0)-a\right| \\
& =\inf _{a \in \mathbf{C}} \limsup _{t \rightarrow \infty}\left|\int_{t-\delta}^{t}\left(\alpha(s)-\alpha(0)-\delta^{-1} a\right) d s\right| \leq \delta M .
\end{aligned}
$$


Thus

$$
G \leq\left|\frac{\eta \delta}{1-e^{-i \eta \delta}}\right|\left\{2\left(\frac{2}{\pi} \min (\mu, 1)+\mu\right) M+\lim \sup _{t \rightarrow \infty} \sup _{t-\delta \leq s \leq t}|\alpha(s)-\alpha(t)|\right\} .
$$

As $\delta \downarrow 0$,

so

$$
\left|\frac{\eta \delta}{1-e^{-i \eta \delta}}\right| \rightarrow 1
$$

$$
G \leq 2\left(\frac{2}{\pi} \min (\mu, 1)+\mu\right) M+L .
$$

In the case when $\eta=0,(3.6)$ is replaced by

$$
\int_{0}^{t} f_{\delta}(s) d s=\delta\left(g_{t}(0)-\alpha(t)\right)+\int_{t-\delta}^{t} \alpha(s) d s,
$$

and the result follows in a similar way.

In the case when $E$ is empty, the first statement of Theorem 2.2 may be applied to show that the limsup in (3.8) is zero, and it follows that $G \leq L$.

Proof of Theorem 2.4. To prove the modified versions of Theorems 2.1 and 2.2 , one incorporates the additional ideas of Lemma 3.1 and Theorem 4.1 of [2] into the proofs given above. Since the choice of $\lambda$, and therefore of $\xi_{ \pm}$, is now determined by $\mu^{\prime}$, and hence by $E^{\prime}$, instead of $\mu$ and $E$, the path $\gamma_{2}$ is in each case modified by including a finite number of semicircles in the right half-plane whose diameters are intervals in the imaginary axis whose total length is arbitrarily small and which cover all points of $E$ lying between the endpoints of $\gamma_{2}$ (or one of its constituent parts). This ensures that $g$ is analytic throughout the interior of the contour. The integrand is modified by including for each interval $\left(\xi_{j}-\varepsilon_{j}, \xi_{j}+\varepsilon_{j}\right)$ a factor $h_{j}(z)$ of the form

$$
h_{j}(z)=\left(1+\frac{\varepsilon_{j}^{2}}{\left(z-i \xi_{j}\right)^{2}}\right) \frac{\left(\eta-\xi_{j}\right)^{2}}{\left(\eta-\xi_{j}\right)^{2}+\varepsilon_{j}^{2}} .
$$

The estimates are very similar to those given in [2], and the details are left to the reader.

The modified version of Theorem 2.3 follows from that of 2.2 , provided that it can be shown that

$$
\sup _{y \in E \backslash E^{\prime}} \sup _{l>0}\left|\int_{0}^{t} e^{-i y s} f_{\delta}(s) d s\right|<\infty,
$$

where $f_{\delta}$ is defined by (3.5). As in the proofs of Theorems 2.1 and 2.2, we may assume that $\sup _{|s-t| \leq \delta}|\alpha(s)-\alpha(t)|<\infty$. By (3.7),

$$
\left|\int_{0}^{t} e^{-i y s} f_{\delta}(s) d s\right| \leq \delta\left(\left|g_{t}(i y)\right|+\sup _{t-\delta \leq s \leq t}|\alpha(s)-\alpha(t)|\right)
$$

and this is bounded, independently of $t$ and $y$, by the assumptions on $\alpha$. 
Proof of Corollary 2.5. Theorem 2.1 may be applied with $\alpha$ replaced by $\alpha_{\zeta}$ (where $\alpha_{\zeta}(t)=g_{t}(i \zeta)$, so that $M=0$ ) and $\eta$ replaced by $\eta+\zeta$.

Proof of Corollary 2.6. This is the modified version of Theorem 2.3 in the case that $E^{\prime}$ is empty and $L=0$. Alternatively, Corollary 2.6 may be deduced by applying the corresponding Tauberian theorem for Laplace transforms of bounded functions [2, Theorem 4.1] to the functions $t \mapsto e^{-i \eta t} f_{\delta}(t)$, where $f_{\delta}$ is defined by (3.5).

\section{LAPLACE TRANSFORMS}

Suppose that $\alpha$ is locally absolutely continuous and eventually Lipschitz, so that (2.2), (2.3), and (2.4) hold, where $f$ is eventually bounded, and $N=$ $\lim \sup _{t \rightarrow \infty}|f(t)|$. Moreover,

$$
G=\limsup _{t \rightarrow \infty}\left|\int_{0}^{t} e^{-i \eta s} f(s) d s-g(i \eta)\right| .
$$

Replacing $f(s)$ by $e^{-i \eta s} f(s)$, we see that estimates on $G$ show how close $f$ is to being conditionally integrable. Theorem 2.2 gives $G \leq 2 N / d$, while Ingham's estimate led to $G \leq 6 \mathrm{~N} / d$ (see $\S 2$ ). If we know that $\alpha$ is eventually bounded, we can obtain another estimate on $G$ from Theorem 2.2.

A further estimate may be obtained by adapting the techniques of $[8$, Theorem 2.1], as follows.

Proposition 4.1. If $\alpha$ is Lipschitz on $[\tau, \infty)$ for some $\tau>0$, then

$$
G \leq N\left(\frac{1}{2}|a+b|+\left(\frac{2}{\pi}+1\right)(b-a)\right) .
$$

where $a=\inf _{y \in E}(y-\eta)^{-1}, b=\sup _{y \in E}(y-\eta)^{-1}(a=b=0$ if $E$ is empty).

Proof. As in the proof of Theorem 2.1, we may assume that $\alpha(t)=\int_{0}^{t} f(s) d s$, where $\lim \sup _{s \rightarrow \infty}|f(s)|=N$. Let $\delta>0, \sigma \in \mathbf{R}$, and put

$$
f_{\delta \sigma}= \begin{cases}e^{-i(\sigma+\eta) t} f(t) & (0 \leq t<\delta), \\ e^{-i(\sigma+\eta) t} f(t)-e^{-i(\sigma+\eta)(t-\delta)} f(t-\delta) & (t \geq \delta) .\end{cases}
$$

The Laplace transform of $f_{\delta \sigma}$ is

$$
\begin{aligned}
g_{\delta \sigma}(z) & =\int_{0}^{\infty} e^{-(z+i \eta+i \sigma) t} f(t) d t-e^{-\delta z} \int_{\delta}^{\infty} e^{-(z+i \eta+i \sigma)(t-\delta)} f(t) d t \\
& =\left(1-e^{-\delta z}\right) g(z+i \eta+i \sigma) .
\end{aligned}
$$

The singular set of $g_{\delta \sigma}$ is $i E_{1}$, where $E_{1} \subseteq E-(\eta+\sigma)$. Moreover,

$$
\begin{aligned}
\underset{t \rightarrow \infty}{\limsup } & \left|\int_{0}^{t} f_{\delta \sigma}(s) d s\right| \\
= & \limsup _{t \rightarrow \infty}\left|\int_{0}^{t} e^{-i(\sigma+\eta) s} f(s) d s-\int_{\delta}^{t} e^{-i(\sigma+\eta)(s-\delta)} f(s-\delta) d s\right| \\
& =\limsup _{t \rightarrow \infty}\left|\int_{t-\delta}^{t} e^{-i(\sigma+\eta) s} f(s) d s\right| \leq N \delta .
\end{aligned}
$$


Now Theorem 2.2 gives

$$
\limsup _{t \rightarrow \infty}\left|\int_{0}^{t} e^{i \sigma s} f_{\delta \sigma}(s) d s-g_{\delta \sigma}(-i \sigma)\right| \leq\left(\frac{2}{\pi}+1\right) 2 N \delta \mu_{\sigma},
$$

where

$$
\begin{aligned}
\mu_{\sigma}=\sup _{y \in E_{1}}\left|\frac{y}{y+\sigma}\right| & \leq \sup _{y \in E}\left|\frac{y-(\sigma+\eta)}{y-\eta}\right| \\
& =\sup _{y \in E}\left|1-\frac{\sigma}{y-\eta}\right|=\max (|1-\sigma a|,|1-\sigma b|) .
\end{aligned}
$$

But

$$
\begin{aligned}
\int_{0}^{t} e^{i \sigma s} f_{\delta \sigma}(s) d s & =\int_{0}^{t} e^{-i \eta s} f(s) d s-e^{i \sigma \delta} \int_{\delta}^{t} e^{-i \eta(s-\delta)} f(s-\delta) d s \\
& =\left(1-e^{i \sigma \delta}\right) \int_{0}^{t} e^{-i \eta s} f(s) d s+e^{i \sigma \delta} \int_{t-\delta}^{t} e^{-i \eta s} f(s) d s .
\end{aligned}
$$

Thus, if $\sigma \delta / 2 \pi \notin \mathbf{Z}$,

$$
G \leq 2\left(\frac{2}{\pi}+1\right) N \frac{\delta \mu_{\sigma}}{\left|1-e^{i \sigma \delta}\right|}+\frac{N \delta}{\left|1-e^{i \sigma \delta}\right|} .
$$

Letting $\delta \downarrow 0$ gives:

$$
G \leq \frac{N}{|\sigma|}\left(2\left(\frac{2}{\pi}+1\right) \mu_{\sigma}+1\right) \quad(\sigma \neq 0) .
$$

Now we put $\sigma=2(a+b)^{-1}$. (If $a+b=0$, we use an approximation argument, letting $\sigma \rightarrow \infty$.) Then

$$
\begin{gathered}
\mu_{\sigma}=\max \left(\left|1-\frac{2 a}{a+b}\right|,\left|1-\frac{2 b}{a+b}\right|\right)=\frac{b-a}{|a+b|}, \\
G \leq N \frac{|a+b|}{2}\left(2\left(\frac{2}{\pi}+1\right) \frac{b-a}{|a+b|}+1\right) \\
=N\left(\frac{1}{2}|a+b|+\left(\frac{2}{\pi}+1\right)(b-a)\right) .
\end{gathered}
$$

If, in obtaining (4.2), we had used the alternative estimate in Theorem 4.2, the subsequent argument would have given the sharpest estimate by letting $\sigma \rightarrow \infty$, and we would have recovered (2.5). To compare the estimates (2.5) and (4.1), one should note that $1 / d=\max (b,-a)$.

Example. Let $\alpha(t)=t, \eta \neq 0$. Then $g(z)=1 / z, E=\{0\}, N=1, a=b=$ $-\eta^{-1}, d=|\eta|$, and

$$
\left|g_{t}(i \eta)-g(i \eta)\right|=\left|\frac{i e^{-i \eta t}}{\eta}\right|=\frac{1}{|\eta|} .
$$

The right-hand side of $(2.5)$ is $2|\eta|^{-1}$, while the right-hand side of (4.1) is $|\eta|^{-1}$. 
When $\alpha$ is Lipschitz, (2.7) is automatically satisfied, and Corollary 2.6 reduces to the Tauberian theorem for Laplace transforms of bounded functions given in [2, Theorem 4.1]. An alternative theorem, proved in a similar way [2, Theorem 4.4], showed that $G=0$ if $\alpha$ is Lipschitz, $E$ is null, and, for each $y$ in $E, g$ is bounded on $\left\{z \in \mathrm{C}: \operatorname{Re} z>0,|z-i y|<\delta_{y}\right\}$ for some $\delta_{y}>0$. We take this opportunity to acknowledge that this result could have been obtained by a careful study of Ingham's method [3].

\section{POWER SERIES}

Let $h(z)=\sum_{n=0}^{\infty} a_{n} z^{n}$ be a power series with radius of convergence 1 , and put

$$
\alpha(0)=0, \quad \alpha(t)=\sum_{n=0}^{m} a_{n} \quad(m<t \leq m+1)
$$

Then

$$
g(z)=\sum_{n=0}^{\infty} a_{n} e^{-n z}=h\left(e^{-z}\right) \quad(\operatorname{Re} z \geq 0) .
$$

Let $F$ be the singular set of $h$ in the unit circle $\Gamma$. Then

$$
E=\left\{y \in \mathbf{R}: e^{-i y} \in F\right\} \text {. }
$$

Take $\lambda=e^{-i \eta} \in \Gamma \backslash F$. Then

$$
\mu=\sup _{y \in E}\left|\frac{y}{y-\eta}\right|=\sup _{e^{-i y} \in F}\left|\frac{y}{y-\eta}\right| \geq 1
$$

since $E$ is nonempty and periodic.

Theorem 2.1 shows that if the partial sums $\left(\sum_{n=0}^{m} a_{n}\right)$ are bounded, then

$$
\underset{m \rightarrow \infty}{\limsup }\left|\sum_{n=0}^{m} a_{n} \lambda^{n}-h(\lambda)\right| \leq 2\left(\frac{2}{\pi}+\mu\right) M,
$$

where

$$
M=\inf _{a \in \mathbf{C}} \limsup _{m \rightarrow \infty}\left|\sum_{n=0}^{m} a_{n}-a\right| .
$$

Ransford [8, Theorem 1.1] showed that

$$
\limsup _{m \rightarrow \infty}\left|\sum_{n=0}^{m} a_{n} \lambda^{n}-h(\lambda)\right| \leq 2 M^{*} \nu,
$$

where

$$
M^{*}=\limsup _{l, m \rightarrow \infty}\left|\sum_{n=l}^{m} \frac{a_{n}}{n}\right|, \quad \nu=\sup _{z \in F}\left|\frac{z-1}{z-\lambda}\right| .
$$

The important qualitative difference between (5.2) and (5.3) is that $\nu$ may take any nonnegative value (depending on the relative positions of $1, \lambda$, and the 
points of $F$ ), but the periodicity forces $\mu \geq 1$. Theorem 2.1 took no account of the periodicity which was built into the techniques of [8].

If $\left(a_{n}\right)$ is bounded, then $L=\lim \sup _{n \rightarrow \infty}\left|a_{n}\right|$. Ransford [8, Theorem 2.1] showed that

$$
\limsup _{m \rightarrow \infty}\left|\sum_{n=0}^{m} a_{n} \lambda^{n}-h(\lambda)\right| \leq \frac{L}{2}\left\{\left(1+\left(\frac{a^{\prime}+b^{\prime}}{2}\right)^{2}\right)^{1 / 2}+2\left(b^{\prime}-a^{\prime}\right)\right\},
$$

where

$$
a^{\prime}=\inf _{z \in F} \operatorname{Im}\left(\frac{z+\lambda}{z-\lambda}\right), \quad b^{\prime}=\sup _{z \in F} \operatorname{Im}\left(\frac{z+\lambda}{z-\lambda}\right) .
$$

This is analogous to Proposition 4.1.

In its stated form, Corollary 2.6 is applicable to power series only if we know, a priori, that $a_{n} \rightarrow 0$. But then a classical theorem of Riesz $[9,7.31]$ implies immediately that $\sum a_{n} \lambda^{n}$ converges to $h(\lambda)$ at all regular points $\lambda$. If we do not know that $a_{n} \rightarrow 0$, then we can deduce Theorem 4 of [1] from the analogous Laplace transform result [2, Theorem 4.1] by the method used to prove Theorem 2.3. Put

$$
\begin{aligned}
\tilde{f}(t) & =a_{m} \quad(m<t \leq m+1), \\
\tilde{\alpha}(t)=\int_{0}^{t} \tilde{f}(s) d s & =\sum_{n=0}^{m-1} a_{n}+(t-m) a_{m} \quad(m<t \leq m+1) .
\end{aligned}
$$

Then

$$
\tilde{g}(z)=\int_{0}^{\infty} e^{-z t} f(t) d t=\left(\frac{1-e^{-z}}{z}\right) h\left(e^{-z}\right) .
$$

The condition

$$
\sup _{\zeta \in F} \sup _{N}\left|\sum_{n=0}^{N} a_{n} \zeta^{n}\right|<\infty
$$

implies that $\left(a_{n}\right)$ is bounded ( $F$ is nonempty), so $\tilde{f}$ is bounded, and

$$
\sup _{y \in E} \sup _{t>0}\left|\int_{0}^{t} e^{-i y s} \tilde{f}(s) d s\right|<\infty .
$$

Moreover, $E$ is null if $F$ is null. Under these conditions, [2, Theorem 4.1] gives

$$
\sum_{n=0}^{N} a_{n}=\int_{0}^{N+1} f(t) d t \rightarrow g(0)=h(1) \quad \text { as } N \rightarrow \infty .
$$

It is possible to modify Corollary 2.6 by assuming in (2.7) and (2.8) that $t \rightarrow \infty$ through a sequence of values. This version of Corollary 2.6 does imply Theorem 4 of [1]. 


\section{DIRICHLET SERIES}

Let $\left(a_{n}\right)_{n \geq 1}$ be any sequence, and put

$$
\alpha(0)=0, \quad \alpha(t)=\sum_{n=1}^{m} \frac{a_{n}}{n} \quad(\log m<t \leq \log (m+1)) .
$$

Then

$$
\begin{aligned}
g(z) & =\sum_{n=1}^{\infty} \frac{a_{n}}{n^{1+z}}, \\
|\alpha(s)-\alpha(t)|=\left|\sum_{e^{s} \leq n<e^{t}} \frac{a_{n}}{n}\right| & \leq\left(\sup _{e^{s} \leq n<e^{t}}\left|a_{n}\right|\right)\left(\int_{e^{s}}^{e^{t}} \frac{d u}{u}+e^{-s}\right) \\
& =\left(\sup _{e^{s} \leq n<e^{t}}\left|a_{n}\right|\right)\left(t-s+e^{-s}\right) .
\end{aligned}
$$

If $\left(a_{n}\right)$ is bounded, it follows that $L=0$.

For each regular point $i \eta$, the results of $\S 2$ have the following consequences for Dirichlet series.

Theorem 6.1. If $\left(\sum_{n=1}^{m} a_{n} / n\right)_{m \geq 1}$ is bounded, then

$$
\limsup _{m \rightarrow \infty}\left|\sum_{n=1}^{m} \frac{a_{n}}{n^{1+i \eta}}-g(i \eta)\right| \leq 2\left(\frac{2}{\pi}+\max (\mu, 1)\right) M,
$$

where

$$
M=\inf _{a \in \mathbf{C}} \limsup _{m \rightarrow \infty}\left|\sum_{n=1}^{m} \frac{a_{n}}{n}-a\right|, \quad \mu=\sup _{y \in E}\left|\frac{y}{y-\eta}\right| .
$$

Theorem 6.2. If $\left(a_{n}\right)_{n \geq 1}$ is bounded and $\left(\sum_{n=1}^{m} a_{n} / n\right)_{m \geq 1}$ is bounded, then

$$
\limsup _{m \rightarrow \infty}\left|\sum_{n=1}^{m} \frac{a_{n}}{n^{1+i \eta}}-g(i \eta)\right| \leq 2\left(\frac{2}{\pi} \min (\mu, 1)+\mu\right) M .
$$

Theorem 6.3. If $\left(a_{n}\right)_{n \geq 1}$ is bounded, $E$ is null, and

$$
\sup _{y \in E} \sup _{m \geq 1}\left|\sum_{n=1}^{m} \frac{a_{n}}{n^{1+i y}}\right|<\infty,
$$

then

$$
\sum_{n=1}^{m} \frac{a_{n}}{n^{1+i \eta}} \rightarrow g(i \eta) \quad \text { as } m \rightarrow \infty
$$

for all $\eta$ in $\mathbf{R} \backslash E$.

In the notation of Remark 8 at the end of $\S 2$, it is not difficult to see that

$$
\Theta_{x} \leq \frac{1}{x}\left(\limsup _{n \rightarrow \infty}\left|a_{n}\right|\right) \text {. }
$$


Thus Ingham's Theorem gives, on letting $x \rightarrow \infty$,

$$
\limsup _{m \rightarrow \infty}\left|\sum_{n=1}^{m} \frac{a_{n}}{n^{1+i \eta}}-g(i \eta)\right| \leq \frac{6}{d} \limsup _{n \rightarrow \infty}\left|a_{n}\right| .
$$

In the case when $E$ is empty, $d$ may be taken arbitrarily large, and the conclusion of Theorem 6.3 follows (see also [7]).

Our results may be applied to more general Dirichlet series by taking $\alpha$ to have steps at points other than $t=\log n$.

\section{7. $C_{0}$-SEMigroups}

Let $\mathscr{T}=\{T(t): t \geq 0\}$ be a $C_{0}$-semigroup on a complex Banach space $X$, with generator $A$, and let $S_{1}$ and $S_{2}$ be bounded linear operators on $X$. We shall suppose that

$$
\sigma(A) \subseteq\{\lambda \in \mathbf{C}: \operatorname{Re} \lambda \leq 0\} .
$$

Suppose, for simplicity, that $\alpha$ is of locally bounded variation, and that

$$
\int_{0}^{\infty}\|T(t)\| d v(t)<\infty
$$

where $v(t)$ is the total variation of $\alpha$ on $[0, t]$. We may then define a bounded linear operator $g(-A)$ by

$$
g(-A)=\int_{0}^{\infty} T(t) d \alpha(t)
$$

This notation is consistent with the use of $g$ as the Laplace-Stieltjes transform of $\alpha$ and with the conventions concerning semigroups in the sense described by the following examples.

If $\alpha(t)=-e^{-\lambda t} / \lambda$, where $\operatorname{Re} \lambda>0$, then

$$
\begin{gathered}
g(z)=\int_{0}^{\infty} e^{-\lambda t} e^{-z t} d t=(\lambda+z)^{-1}, \\
g(-A)=\int_{0}^{\infty} e^{-\lambda t} T(t) d t=(\lambda-A)^{-1} .
\end{gathered}
$$

If

then

$$
\alpha(t)= \begin{cases}0 & \left(t \leq t_{0}\right) \\ 1 & \left(t>t_{0}\right)\end{cases}
$$

$$
g(z)=e^{-t_{0} z}, \quad g(-A)=T\left(t_{0}\right)=e^{t_{0} A} .
$$

The mapping $g \mapsto g(-A)$ is an algebra isomorphism from the algebra of Laplace-Stieltjes transforms $g$ arising from suitable $\alpha$ (satisfying (7.1)) into the algebra of bounded linear operators on $X$.

If $\alpha$ is given by (5.1), then

$$
g(z)=\sum_{n=0}^{\infty} a_{n} e^{-n z}, \quad g(-A)=\sum_{n=0}^{\infty} a_{n} T(1)^{n} .
$$

Thus the following theorem is an analogue of [1, Theorem 5]. 
Theorem 7.1. Suppose that $\sup _{t>0}\left\|S_{1} T(T) S_{2}\right\|<\infty, \int_{0}^{\infty} t d v(t)<\infty$, and $g(-z)=0$ for all $z$ in $\sigma(A) \cap i \mathbf{R}$. Then

$$
\left\|S_{1} g(-A)\left(\int_{0}^{t} e^{-i \eta s} T(s) d s-(i \eta-A)^{-1}\right) S_{2}\right\| \rightarrow 0
$$

as $t \rightarrow \infty$, for all $\eta$ in $\mathbf{R}$ with in $\notin \sigma(A)$.

Proof. Let $E_{1}=\{\eta \in \mathbf{R}: i \eta \in \sigma(A)\}$. If $g$ is identically zero, then $g(-A)=0$ and the result is trivial. Otherwise, since $g$ is the Laplace-Stieltjes transform of a function of bounded variation, its zeros on the imaginary axis form a null set, so $E_{1}$ is null.

Let

$$
f(t)=S_{1} g(-A) T(t) S_{2}=\int_{0}^{\infty} S_{1} T(s+t) S_{2} d \alpha(s) .
$$

Then $f$ is bounded, with Laplace transform

$$
\begin{aligned}
\int_{0}^{\infty} e^{-z t} f(t) d t & =S_{1}\left(\int_{0}^{\infty} T(s) d \alpha(s)\right)\left(\int_{0}^{\infty} e^{-z t} T(t) d t\right) S_{2} \\
& =S_{1} g(-A)(z-A)^{-1} S_{2} .
\end{aligned}
$$

Thus the singular set is contained in $i E_{1}$-in particular it is null.

For $\eta$ in $E_{1}$,

$$
\alpha_{-\eta}(t)=\int_{0}^{t} e^{i \eta s} d \alpha(s)=-\int_{t}^{\infty} e^{i \eta s} d \alpha(s)
$$

since $g(-i \eta)=0$. Now

$$
\begin{aligned}
& \int_{0}^{t} e^{-i \eta s} f(s) d s \\
&=\int_{0}^{t} \int_{0}^{\infty} e^{-i \eta s} S_{1} T(s+u) S_{2} d \alpha(u) d s \\
&=\int_{0}^{\infty} \int_{0}^{t} e^{-i \eta(s+u)} S_{1} T(s+u) S_{2} d s e^{i \eta u} d \alpha(u) \\
&=\int_{0}^{\infty} \int_{u}^{u+t} e^{-i \eta s} S_{1} T(s) S_{2} d s e^{i \eta u} d \alpha(u) \\
&=\lim _{t^{\prime} \rightarrow \infty} \int_{t^{\prime}}^{t^{\prime}+t} e^{-i \eta s} S_{1} T(s) S_{2} d s \alpha_{-\eta}\left(t^{\prime}\right) \\
&-\lim _{t^{\prime} \rightarrow \infty} \int_{0}^{t^{\prime}}\left(e^{-i \eta(u+t)} S_{1} T\left(u+t^{\prime}\right) S_{2}-e^{-i \eta u} S_{1} T(u) S_{2}\right) \alpha_{-\eta}(u) d u \\
&=-\lim _{t^{\prime} \rightarrow \infty} \int_{0}^{t^{\prime}}\left(e^{-i \eta(u+t)} S_{1} T\left(u+t^{\prime}\right) S_{2}-e^{-i \eta u} S_{1} T(u) S_{2}\right) \alpha_{-\eta}(u) d u
\end{aligned}
$$


using integration by parts and the facts that $S_{1} T(s) S_{2}$ is bounded and $\alpha_{-\eta}\left(t^{\prime}\right) \rightarrow$ 0 as $t^{\prime} \rightarrow \infty$. If $M=\sup _{s>0}\left\|S_{1} T(s) S_{2}\right\|$,

$$
\begin{aligned}
\left\|\int_{0}^{t} e^{-i \eta s} f(s) d s\right\| & \leq 2 M \int_{0}^{\infty}\left|\alpha_{-\eta}(u)\right| d u \\
& \leq 2 M \int_{0}^{\infty} \int_{u}^{\infty} d v(s) d u \leq 2 M \int_{0}^{\infty} s d v(s)
\end{aligned}
$$

which is finite and independent of $t$ and $\eta$. Thus the conditions of [2, Theorem 4.1] are satisfied, and the result follows immediately.

Corollary 7.2. Suppose that $\sup _{t>0}\left\|S_{1} T(t)\right\|<\infty, \int_{0}^{\infty} t d v(t)<\infty$, and $g(-z)$ $=0$ for all $z$ in $\sigma(A) \cap i \mathbf{R}$. Then $S_{1} g(-A) T(t) \rightarrow 0$ strongly as $t \rightarrow \infty$.

Proof. Choose $\eta$ so that $i \eta \notin \sigma(A)$. By Theorem 7.1,

$$
\int_{0}^{t} e^{-i \eta s} S_{1} g(-A) T(s) d s
$$

converges in norm and hence strongly as $t \rightarrow \infty$. But

$$
\begin{aligned}
& \left\|e^{-i \eta(s+u)} S_{1} g(-A) T(s+u) x-e^{-i \eta s} S_{1} g(-A) T(s) x\right\| \\
& \quad \leq\left\|S_{1} g(-A) T(s)\right\|\left\|e^{-i \eta u} T(u) x-x\right\| \leq M\|g(-A)\|\left\|e^{-i \eta u} T(u) x-x\right\|,
\end{aligned}
$$

so the integrand is uniformly strongly continuous. Hence the integrand tends to zero strongly.

Suppose that $\int_{0}^{\infty} t d v(t)<\infty, g(-z)=0$ for all $z$ in $\sigma(A) \cap i \mathbf{R}$, and $\sup _{t>0}\left\|S_{1} T(t) x\right\|$ is finite for some $x$. Either by applying Theorem 7.1 with $S_{2}$ an operator of rank one with $S_{2} x=x$, or by repeating the proof of Theorem 7.1 with $f(t)=S_{1} g(-A) T(t) x$, one can see that

$$
\left\|S_{1} g(-A)\left(\int_{0}^{\infty} e^{-i \eta s} T(s) d s-(i \eta-A)^{-1}\right) x\right\| \rightarrow 0
$$

as $t \rightarrow \infty$ for all real $\eta$ with $i \eta \notin \sigma(A)$.

Example. Suppose that $\sigma(A) \cap i \mathbf{R}$ is a finite set, say $\left\{i \eta_{j}: j=1, \ldots, k\right\}$. Let $t_{j}>0, \operatorname{Re} \lambda_{j}>0(j=1, \ldots, k)$,

$$
g_{1}(z)=\prod_{j=1}^{k}\left(e^{-t_{j} z}-e^{i \eta_{j} t_{j}}\right), \quad g_{2}(z)=\prod_{j=1}^{k} \frac{z+i \eta_{j}}{z+\lambda_{j}} .
$$

Then $g_{1}$ and $g_{2}$ are both Laplace-Stieltjes transforms, arising from convolutions of measures given by

and

$$
\alpha_{j}(t)= \begin{cases}0 & (t=0), \\ -e^{i \eta_{j} t_{j}} & \left(0<t \leq t_{j}\right), \\ 1-e^{i \eta_{j} t_{j}} & \left(t>t_{j}\right),\end{cases}
$$

$$
\alpha_{j}(t)= \begin{cases}0 & (t=0), \\ 1+\left(1-i \eta_{j} / \lambda_{j}\right) e^{-\lambda_{j} t} & (t>0),\end{cases}
$$


respectively. Furthermore

$$
\begin{aligned}
& g_{1}(-A)=\prod_{j=1}^{k}\left(T\left(t_{j}\right)-e^{i \eta_{j} t_{j}}\right), \\
& g_{2}(-A)=\prod_{j=1}^{k}\left(i \eta_{j}-A\right)\left(\lambda_{j}-A\right)^{-1}=\prod_{j=1}^{k}\left(1+\left(i \eta_{j}-\lambda_{j}\right)\left(\lambda_{j}-A\right)^{-1}\right) .
\end{aligned}
$$

Corollary 7.2 shows that if $\left\|S_{1} T(t)\right\|$ is bounded, then $S_{1} T(t) g_{r}(-A) \rightarrow 0$ strongly $(r=1,2)$.

If the residual spectrum $R \sigma(A)$ of $A$ contains no point of the imaginary axis $i \mathbf{R}$, then $R \sigma\left(T\left(t_{j}\right)\right)$ contains no point of the unit circle $\Gamma$, and $\left(\lambda_{j}-i \eta_{j}\right)^{-1} \notin$ $R \sigma\left(\left(\lambda_{j}-A\right)^{-1}\right)$. It follows that $g_{r}(-A)$ has dense range $(r=1,2)$, and hence that $S_{1} T(t) \rightarrow 0$ strongly if $\left\|S_{1} T(t)\right\|$ is bounded.

The final conclusion of this example extends to the case when $\sigma(A) \cap i \mathbf{R}$ is countably infinite (and $R \sigma(A) \cap i \mathbf{R}$ is empty). The argument of [2, §3] shows that $S_{1} T(t) \rightarrow 0$ strongly if $\left\|S_{1} T(t)\right\|$ is bounded. Lyubich and Phong [6] have given an independent proof of the stability theorem in [2], but their argument does not seem to extend to the case when $S_{1}$ and $T(t)$ do not commute.

If $\sigma(A) \cap i \mathbf{R}$ is empty and $\left\|S_{1} T(t) S_{2}\right\|$ is bounded, then the Korevaar-Zagier version of Ingham's Theorem (see Theorem 2.2), with $\alpha(t)=S_{1} T(t) A^{-1} S_{2}=$ $\int_{0}^{t} S_{1} T(t) S_{2} d s+S_{1} A^{-1} S_{2}$, gives $\left\|S_{1} T(t) A^{-1} S_{2}\right\| \rightarrow 0$. Nevertheless, it does not follow that $S_{1} T(t) S_{2} \rightarrow 0$ strongly. In the setting of Example 2.5b) of [2], $X=c_{0}, \sup _{t>0}\|T(t) y\|<\infty$ but $\lim \sup _{t \rightarrow \infty}\|T(t) y\|>0$, for some $y$. We may then take $S_{1}=I$ and $S_{2}$ to be an operator of rank one with $S_{2} y=y$. The same example on the Hilbert space $l^{2}$ has similar properties. However, we do have the following result for reflexive spaces.

Proposition 7.3. Suppose that $X$ is reflexive, $\sigma(A) \cap i \mathbf{R}$ is countable, and $\operatorname{P\sigma }(A) \cap i \mathbf{R}$ is empty. Then $T(t) x \rightarrow 0$ weakly for each $x$ such that $\sup _{t>0}\|T(t) x\|<\infty$. Furthermore, there is a net $\left(T_{\alpha}\right)$ in the convex hull of $\{T(t): t \geq 0\}$ such that $\left\|T_{\alpha} x\right\| \rightarrow 0$ for all such $x$.

Proof. The dual semigroup on $X^{*}$ is strongly continuous with generator $A^{*}$, and $\sigma\left(A^{*}\right)=\sigma(A), R \sigma\left(A^{*}\right)=P \sigma(A)$. Let $\phi$ be a nonzero functional in $X^{*}$, and define an operator $S_{1}$ on $X^{*}$ by $S_{1} \psi=\psi(x) \phi$. Then $\left\|S_{1} T(t)^{*}\right\|=$ $\|T(t) x\|\|\phi\|$. Applying the remarks above to the dual semigroup, it follows that $\left\|S_{1} T(t)^{*} \psi\right\| \rightarrow 0$ for all $\psi$, so $\psi(T(t) x) \rightarrow 0$. The final statement is a standard Hahn-Banach argument.

\section{SiNGLE OPERATORS}

Let $T$ be a bounded linear operator on $X$ with spectral radius 1 , and let $S_{1}$ and $S_{2}$ be any bounded linear operators on $X$. From a slight modification of [1, Theorem 5], we can easily obtain the following. 
Proposition 8.1. Suppose that the peripheral spectrum of $T, \sigma(T) \cap \Gamma$, is a finite set $\left\{\lambda_{1}, \ldots, \lambda_{k}\right\}$ and that $\sup _{n}\left\|S_{1} T(t) S_{2}\right\|<\infty$. Then

$$
\left\|S_{1}\left(\lambda_{1}-T\right) \cdots\left(\lambda_{k}-T\right) T^{n} S_{2}\right\| \rightarrow 0 \quad \text { as } n \rightarrow \infty .
$$

Proof. Let

$$
\begin{aligned}
f(z) & =S_{1}\left(\lambda_{1}-T\right) \cdots\left(\lambda_{k}-T\right)(1-z T)^{-1} S_{2} \\
& =\sum_{n=0}^{\infty} S_{1}\left(\lambda_{1}-T\right) \cdots\left(\lambda_{k}-T\right) T^{n} S_{2} z^{n} \quad(|z| \leq 1) .
\end{aligned}
$$

As in the proof of [1, Theorem 5], it is easily verified that (8.2) converges in norm for any $z$ in $\Gamma \backslash \sigma(T)$. Now (8.1) follows.

We may also obtain the following analogues of results of $\S 7$.

1. If $\sigma(T) \cap \Gamma$ is countable, $R \sigma(T) \cap \Gamma$ is empty, and $\sup _{n}\left\|S_{1} T^{n}\right\|<\infty$, then $S_{1} T^{n} \rightarrow 0$ strongly.

2. There is an operator $T$ on $l^{2}$ with spectral radius $1, \sigma(T) \cap \Gamma=\{1\}$, $1 \notin R \sigma(T) \cup P \sigma(T)$, and a vector $x$ in $l^{2}$ such that $0<\limsup _{n \rightarrow \infty}\left\|T^{n} x\right\|<$ $\infty$.

3. Suppose that $X$ is reflexive, $\sigma(T) \cap \Gamma$ is countable, and $P \sigma(T) \cap \Gamma$ is empty. Then $T^{n} x \rightarrow 0$ weakly for each $x$ such that $\sup _{n}\left\|T^{n} x\right\|<\infty$. Furthermore, there is a net $\left(T_{\alpha}\right)$ in the convex hull of $\left\{T^{n}\right\}$ such that $\left\|T_{\alpha} x\right\| \rightarrow$ 0 for all such $x$.

\section{REFERENCES}

1. G. R. Allan, A. G. O'Farrell, and T. J. Ransford, A Tauberian theorem arising in operator theory, Bull. London Math. Soc. 19 (1987), 537-545.

2. W. Arendt and C. J. K. Batty, Tauberian theorems and stability of one-parameter semigroups, Trans. Amer. Math. Soc. 306 (1988), 837-852.

3. A. E. Ingham, On Wiener's method in Tauberian theorems, Proc. London Math. Soc. (2) 38 (1935), 458-480.

4. Y. Katznelson and L. Tzafriri, On power bounded operators, J. Funct. Anal. 68 (1986), 313-328.

5. J. Korevaar, On Newman's quick way to the prime number theorem, Math. Intelligencer 4 (1982), 108-115.

6. Y. I. Lyubich and Vu Quoc Phong, Asymptotic stability of linear differential equations in Banach spaces, Studia Math. 88 (1988), 37-42.

7. D. J. Newman, Simple analvtic proof of the prime number theorem, Amer. Math. Monthly 87 (1980), 693-696.

8. T. J. Ransford, Some quantitative Tauberian theorems for power series, Bull. London Math. Soc. 20 (1988), 37-44.

9. E. C. Titchmarsh, The theory of functions, Oxford Univ. Press, Oxford, 1932.

10. D. V. Widder, An introduction to transform theory. Academic Press, New York, 1971.

11. D. Zagier, Short proof of the prime number theorem. unpublished manuscript.

12. C. J. K. Batty and Vu Quoc Phong. Stability of individual elements under one-parameter semigroups. Trans. Amer. Math. Soc. 322 (1990), 805-818. 